\title{
Color-octet scalar decays to a gluon and an electroweak gauge boson in the Manohar-Wise model
}

\author{
Alper Hayreter $\circledast^{1, *}$ and German Valencia $\circledast^{2, \dagger}$ \\ ${ }^{1}$ Department of Natural and Mathematical Sciences, Ozyegin University, 34794 Istanbul Turkey \\ ${ }^{2}$ School of Physics and Astronomy, Monash University, Wellington Road, Clayton, VIC-3800, Australia
}

(Received 9 October 2020; accepted 3 December 2020; published 28 December 2020)

\begin{abstract}
We present one loop results for the amplitudes giving rise to couplings between a color octet scalar, a gluon, and an electroweak gauge boson. These amplitudes could signal new physics in $\gamma$ jet, $Z$ jet and $W$ jet production at the LHC. We compute the relevant branching ratios and identify regions of parameter space where these decay modes become important. This can happen for scalar masses below the threshold for decay into heavy quark pairs $(t \bar{t}$ and $t \bar{b})$ or for small Yukawa couplings in which case the colored scalars are fermiophobic. In the case of light scalars, $\mathcal{B}(S \rightarrow \gamma g)$ can reach up to $10 \%$ whereas $\mathcal{B}(S \rightarrow Z g)$ can reach a few percent. In the fermiophobic region of parameter space, $\mathcal{B}(S \rightarrow \gamma g)$ and $\mathcal{B}(S \rightarrow Z g)$ can reach up to $72 \%$ and $28 \%$ respectively, whereas $\mathcal{B}(S \rightarrow g g)$ can be $100 \%$. For the charged scalar, the decay mode $\mathcal{B}\left(S^{ \pm} \rightarrow W^{ \pm} g\right)$ can become dominant in both scenarios.
\end{abstract}

DOI: $10.1103 /$ PhysRevD.102.115033

\section{INTRODUCTION}

Many extensions of the standard model (SM) contain colored scalars that give rise to rich phenomenology. These include the sgluons and squarks of supersymmetry and scalar leptoquarks, for example. Multi-Higgs models can also include scalar representations charged under the color group. One compelling example is the Manohar-Wise model (MW) [1] in which scalars transforming as a color octet, electroweak doublet are introduced. This particular representation can couple directly to quarks while respecting minimal flavor violation, thus naturally satisfying constraints from flavor physics.

The MW model contains fourteen new parameters in the scalar potential and an additional four parameters in the Yukawa sector and has been studied at length in the literature. For example, the new scalars can modify the $\mathrm{Hgg}$ coupling at one-loop and alter significantly the Higgs production and decay phenomenology [1-7]. The new scalars also affect precision electroweak measurements $[1,8,9]$ and flavor physics [10-13], and all this leads to constraints on its parameters. In addition to these phenomenological constraints, the parameter space is restricted by theoretical considerations such as unitarity and vacuum

\footnotetext{
*alper.hayreter@ozyegin.edu.tr

german.valencia@monash.edu
}

Published by the American Physical Society under the terms of the Creative Commons Attribution 4.0 International license. Further distribution of this work must maintain attribution to the author(s) and the published article's title, journal citation, and DOI. Funded by SCOAP. stability [14-17]. After taking these constraints into account the MW model can still produce many observable effects at the LHC [18-22].

In this paper we study decay modes of the MW scalars that have not received much attention thus far, namely the one-loop induced processes connecting a scalar to a gluon and an electroweak gauge boson $W^{ \pm}, Z$ or $\gamma$. The MW scalars can be pair produced at tree-level through their QCD couplings and subsequently decay. Most of the time they will decay into pairs of heavy quarks through their Yukawa couplings. The effective couplings we compute in this paper induce decays into electroweak gauge bosons and jets that typically occur at much lower rates. However, there are regions of parameter space where these decay modes become dominant.

The phenomenology of new physics in $\gamma j$ and $Z j$ final states at LHC has received some attention in the literature. It has been recently considered in the context of a pseudoscalar color octet $\pi_{8}$ [23], where it is argued that $\gamma j$, in particular, is a clean channel due to the presence of an energetic photon. References [24,25] have studied the decays of sgluons into $\gamma j$ via squark loops. Early studies of an apparent dijet anomaly reported by CDF [26] considered tree-level processes with color octet scalars resulting in $\gamma j$, $Z j$, or $W^{ \pm} j$ final states $[27,28]$. Very recently there has also been a phenomenological study presenting constraints on $\gamma j$ final states at LHC [29].

This paper is organized as follows. In Sec. II we review the relevant features of the MW model paying particular attention to the sector of the model that is relevant for this study. In Sec. III we present explicit one-loop results for the $S V g$ vertices including quark and scalar loop contributions. 
In Sec. IV we discuss the regions of parameter space where these decay modes can become important. In Sec. V we present numerical results for benchmark points illustrating the branching ratios $\mathcal{B}(S \rightarrow V g)$ that can be reached. A phenomenological study of signals for these modes at LHC is beyond the scope of this paper, but we provide preliminary comments by comparing our one-loop vertices with the recent study of [29].

\section{THE MODEL}

In the MW model, the new scalar field $S$ transforms as $(8,2,1 / 2)$ under the SM gauge group $S U(3)_{C} \times S U(2)_{L} \times$ $U(1)_{Y}$. Numerous new couplings appear in the scalar potential and in the Yukawa sector. The possible Yukawa couplings reduce to two complex numbers once minimal flavor violation is imposed [1],

$$
\mathcal{L}_{Y}=-\eta_{U} e^{i \alpha_{U}} g_{i j}^{U} \bar{u}_{R i} T^{A} Q_{j} S^{A}-\eta_{D} e^{i \alpha_{D}} g_{i j}^{D} \bar{d}_{R i} T^{A} Q_{j} S^{\dagger A}+\text { H.c. }
$$

Here $Q_{i}$ are the usual left-handed quark doublets, and $S^{a}$ are the new scalars written as $S=S^{a} T^{a} S U(3)$ generators normalized as $\operatorname{Tr}\left(T^{a} T^{b}\right)=\delta^{a b} / 2$. The matrices $g_{i j}^{U, D}$ are the same as the Higgs couplings to quarks, and the overall strength of the interactions is given by $\eta_{U, D}$ along with their phases $\alpha_{U, D}$. The latter introduce charge-parity $(C P)$ violation beyond the SM and contribute for example to the electric dipole moment (EDM) and chromoelectric dipole moment (CEDM) of quarks $[1,9,12,20]$.

The most general renormalizable scalar potential is given in Ref. [1] The new couplings we derive in this work will only depend on the following terms:

$$
\begin{aligned}
V= & \lambda\left(H^{\dagger i} H_{i}-\frac{v^{2}}{2}\right)^{2}+2 m_{s}^{2} \operatorname{Tr} S^{\dagger i} S_{i}+\lambda_{1} H^{\dagger i} H_{i} \operatorname{Tr} S^{\dagger j} S_{j}+\lambda_{2} H^{\dagger i} H_{j} \operatorname{Tr} S^{\dagger j} S_{i} \\
& +\left(\lambda_{3} H^{\dagger i} H^{\dagger j} \operatorname{Tr} S_{i} S_{j}+\lambda_{4} e^{i \phi_{4}} H^{\dagger i} \operatorname{Tr} S^{\dagger j} S_{j} S_{i}+\lambda_{5} e^{i \phi_{5}} H^{\dagger i} \operatorname{Tr} S^{\dagger j} S_{i} S_{j}+\text { H.c. }\right),
\end{aligned}
$$

where $v \sim 246 \mathrm{GeV}$. The number of parameters in Eq. (2) can be further reduced by theoretical considerations: first $\lambda_{3}$ can be chosen to be real by a suitable definition of $S$; custodial $S U(2)$ symmetry implies the relations $2 \lambda_{3}=\lambda_{2}$ (and hence $\left.m_{S^{+}}=m_{I}\right)$ [1], and $\lambda_{4}=\lambda_{5}^{\star}$ [9]; and $C P$ conservation removes all the phases, $\alpha_{U}, \alpha_{D}, \phi_{4}$ and $\phi_{5}$. After symmetry breaking, the Higgs vacuum expectation value in Eq. (2) splits the octet scalar masses as

$$
m_{S^{ \pm}}^{2}=m_{S}^{2}+\lambda_{1} \frac{v^{2}}{4}, \quad m_{S_{R, I}}^{2}=m_{S}^{2}+\left(\lambda_{1}+\lambda_{2} \pm 2 \lambda_{3}\right) \frac{v^{2}}{4} .
$$

In our calculation, the parameters $\lambda_{1,2,3}$ simply control this mass splitting and will be traded for the scalar masses. The triple scalar coupling depends on $\lambda_{4,5}$, and it determines the magnitude of the scalar loop contributions to the $S g g, S g \gamma, S g Z$ and $S^{ \pm} g W^{ \pm}$we compute next. Finally, $\eta_{U, D}$ control respectively the strength of the $S t t$ and $S b b$ interactions.

The effective one-loop couplings of the form $S V g$ can be written in terms of two (dual) field strength tensors $F(\tilde{F})_{R, I}^{V g}$ as

$$
\begin{aligned}
\mathcal{L}_{S g g} & =\frac{\alpha_{s}}{8 \pi v}\left[\left(F_{R}^{g g} G_{\mu \nu}^{A} G^{B \mu \nu}+\tilde{F}_{R}^{g g} \tilde{G}_{\mu \nu}^{A} G^{B \mu \nu}\right) S_{R}^{C}+\left(F_{I}^{g g} G_{\mu \nu}^{A} G^{B \mu \nu}+\tilde{F}_{I}^{g g} \tilde{G}_{\mu \nu}^{A} G^{B \mu \nu}\right) S_{I}^{C}\right] d^{A B C}, \\
\mathcal{L}_{S \gamma g} & =\frac{\sqrt{\alpha \alpha_{s}}}{3 \pi v}\left[\left(F_{R}^{\gamma g} G_{\mu \nu}^{A} A^{\mu \nu}+\tilde{F}_{R}^{\gamma g} \tilde{G}_{\mu \nu}^{A} A^{\mu \nu}\right) S_{R}^{B}+\left(F_{I}^{\gamma g} G_{\mu \nu}^{A} A^{\mu \nu}+\tilde{F}_{I}^{\gamma g} \tilde{G}_{\mu \nu}^{A} A^{\mu \nu}\right) S_{I}^{B}\right] \delta^{A B}, \\
\mathcal{L}_{S Z g} & =\frac{\sqrt{\alpha \alpha_{s}}}{12 \pi v}\left[\left(F_{R}^{Z g} G_{\mu \nu}^{A} Z^{\mu \nu}+\tilde{F}_{R}^{Z g} \tilde{G}_{\mu \nu}^{A} Z^{\mu \nu}\right) S_{R}^{B}+\left(F_{I}^{Z g} G_{\mu \nu}^{A} Z^{\mu \nu}+\tilde{F}_{I}^{Z g} \tilde{G}_{\mu \nu}^{A} Z^{\mu \nu}\right) S_{I}^{B}\right] \delta^{A B}, \\
\mathcal{L}_{S W g} & =\frac{\sqrt{\alpha \alpha_{s}}}{12 \pi v}\left(F^{W g} G_{\mu \nu}^{A} W^{ \pm \mu \nu}+\tilde{F}^{W g} \tilde{G}_{\mu \nu}^{A} W^{ \pm \mu \nu}\right) S^{\mp B} \delta^{A B},
\end{aligned}
$$

where $G_{\mu \nu}^{A}, A_{\mu \nu}, Z_{\mu \nu}$ and $W_{\mu \nu}$ are the gluon, photon, $Z$ and $W$ field strength tensors respectively and $\tilde{G}^{A \mu \nu}=$ $(1 / 2) \epsilon^{\mu \nu \alpha \beta} G_{\alpha \beta}^{A}$.

Explicit one-loop results for these factors in the MW model are presented in the next section. Of these couplings, only $\mathcal{L}_{S g g}$ exists in the literature, and we find a sign difference with that result that we describe below. These effective vertices receive their main contributions from top-quark and color-octet scalar loops. The bottom-quark loop is important only for regions of parameter space where $\left|\eta_{D}\right| \gg\left|\eta_{U}\right|$.

\section{EXPLICIT ONE-LOOP RESULTS IN THE MW MODEL}

We perform the calculation with the aid of a number of software packages. We first implement the model in 


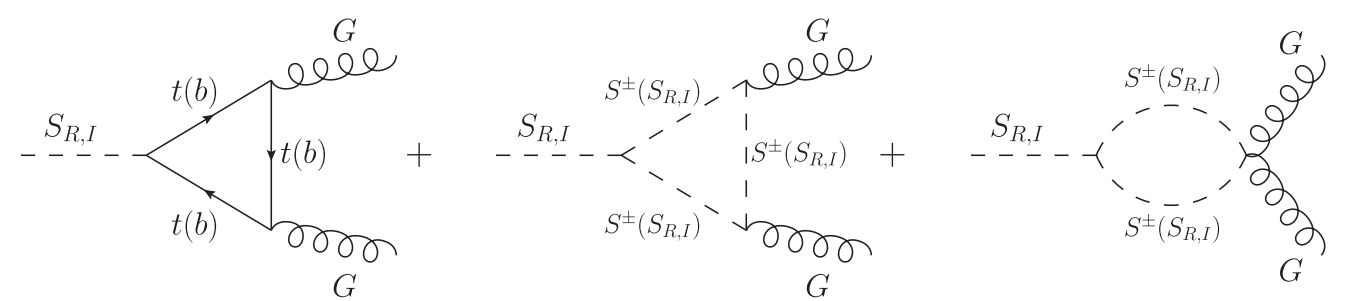

FIG. 1. One-loop diagrams contributing to the factors appearing in Eq. (4) for the $S_{R, I} g g$ coupling.

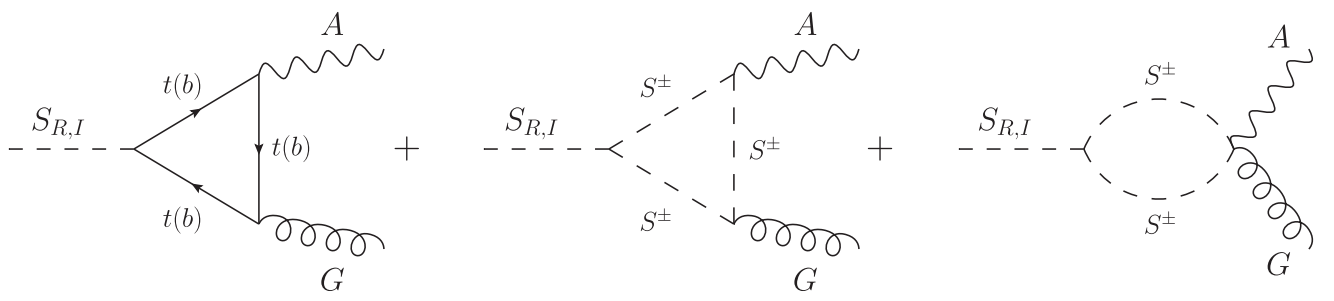

FIG. 2. One-loop diagrams contributing to the factors appearing in Eq. (4) for the $S_{R, I} \gamma g$ coupling.

FeynRules [30,31] to generate FeynArts [32] output where LoopTools [33] is used to compute the one loop diagrams, and simplification is assisted with FeynCalc [34,35], FeynHelpers [36] and Package-X [37]. We present our result without assuming custodial or $C P$ symmetries.

\section{A. $S_{R, I} \rightarrow g g$}

The diagrams responsible for the $S g g$ couplings are shown in Fig. 1 and result in form factors given by

$$
\begin{aligned}
F_{R}^{g g}= & \left\{\eta_{U} c_{U} I_{q}\left(\frac{m_{t}^{2}}{m_{R}^{2}}\right)+\eta_{D} c_{D} I_{q}\left(\frac{m_{b}^{2}}{m_{R}^{2}}\right)\right. \\
& +\frac{9}{4} \frac{v^{2}}{m_{R}^{2}}\left(\lambda_{4} c_{4}+\lambda_{5} c_{5}\right) \\
& \left.\times \frac{1}{2}\left[I_{s}(1)+\frac{1}{3} I_{s}\left(\frac{m_{I}^{2}}{m_{R}^{2}}\right)+\frac{2}{3} I_{s}\left(\frac{m_{S^{ \pm}}^{2}}{m_{R}^{2}}\right)\right]\right\} \\
F_{I}^{g g}= & \left\{-\eta_{U} s_{U} I_{q}\left(\frac{m_{t}^{2}}{m_{I}^{2}}\right)+\eta_{D} s_{D} I_{q}\left(\frac{m_{b}^{2}}{m_{I}^{2}}\right)\right. \\
& -\frac{9}{4} \frac{v^{2}}{m_{I}^{2}}\left(\lambda_{4} s_{4}+\lambda_{5} s_{5}\right) \\
& \left.\times \frac{1}{2}\left[I_{s}(1)+\frac{1}{3} I_{s}\left(\frac{m_{R}^{2}}{m_{I}^{2}}\right)+\frac{2}{3} I_{s}\left(\frac{m_{S^{ \pm}}^{2}}{m_{I}^{2}}\right)\right]\right\} \\
\tilde{F}_{R}^{g g}=[ & \left.-\eta_{U} s_{U} \frac{m_{t}^{2}}{m_{R}^{2}} f\left(\frac{m_{t}^{2}}{m_{R}^{2}}\right)-\eta_{D} s_{D} \frac{m_{b}^{2}}{m_{R}^{2}} f\left(\frac{m_{b}^{2}}{m_{R}^{2}}\right)\right] \\
\tilde{F}_{I}^{g g}=[ & \left.-\eta_{U} c_{U} \frac{m_{t}^{2}}{m_{I}^{2}} f\left(\frac{m_{t}^{2}}{m_{I}^{2}}\right)+\eta_{D} c_{D} \frac{m_{b}^{2}}{m_{I}^{2}} f\left(\frac{m_{b}^{2}}{m_{I}^{2}}\right)\right],
\end{aligned}
$$

where $I_{q}, I_{s}$ and $f$ are familiar from $H g g$ effective couplings and are given below.

Imposing custodial symmetry, the scalar loops only contribute to an $S_{R} g g$ coupling through $F_{R}^{g g}$. Imposing $C P$ symmetry $S_{R}\left(S_{I}\right)$ are pure scalar (pseudoscalar), and therefore only the factors $F_{R}^{g g}$ and $\tilde{F}_{I}^{g g}$ are nonzero. The bottomquark loops are much suppressed with respect to the topquark loops unless $\eta_{D} \gg \eta_{U}$. In the limit of $C P$ conservation and $m_{b}=0$, these results agree with [8] except for the sign in front of the factor $\frac{9}{4}$. This sign, however, is of no consequence for phenomenology as $\lambda_{4,5}$ can have either sign. ${ }^{1}$

$$
\text { B. } S_{R, I} \rightarrow \gamma g
$$

The diagrams leading to $S \gamma g$ effective vertices are shown in Fig. 2, and the resulting form factors are given by

$$
\begin{aligned}
F_{R}^{\gamma g}= & {\left[\eta_{U} c_{U} I_{q}\left(\frac{m_{t}^{2}}{m_{R}^{2}}\right)-\eta_{D} c_{D} \frac{1}{2} I_{q}\left(\frac{m_{b}^{2}}{m_{R}^{2}}\right)\right.} \\
& \left.-\frac{9}{4} \frac{v^{2}}{m_{R}^{2}}\left(\lambda_{4} c_{4}-\lambda_{5} c_{5}\right) \frac{1}{2} I_{s}\left(\frac{m_{S^{ \pm}}^{2}}{m_{R}^{2}}\right)\right] \\
F_{I}^{\gamma g}= & {\left[-\eta_{U} s_{U} I_{q}\left(\frac{m_{t}^{2}}{m_{I}^{2}}\right)-\eta_{D} s_{D} \frac{1}{2} I_{q}\left(\frac{m_{b}^{2}}{m_{I}^{2}}\right)\right.} \\
& \left.+\frac{9}{4} \frac{v^{2}}{m_{I}^{2}}\left(\lambda_{4} s_{4}-\lambda_{5} s_{5}\right) \frac{1}{2} I_{S}\left(\frac{m_{S^{ \pm}}^{2}}{m_{I}^{2}}\right)\right]
\end{aligned}
$$

\footnotetext{
${ }^{1}$ When $C P$ violation is included we find the following errors in [20]: the factors $\tilde{F}_{R, I}^{g g}$ are a factor of 2 too large in [20]; the function $I_{s}(z)$ in Eq. (2.6) of [20] contains an incorrect overall factor of $z$ which is inconsequential in the limit of degenerate scalars. There is also a typo in Eq. (6) of [22], where there should be a minus sign in the term with $\eta_{U}$ in $F_{I}$, corresponding to $\tilde{F}_{I}^{g g}$ here.
} 


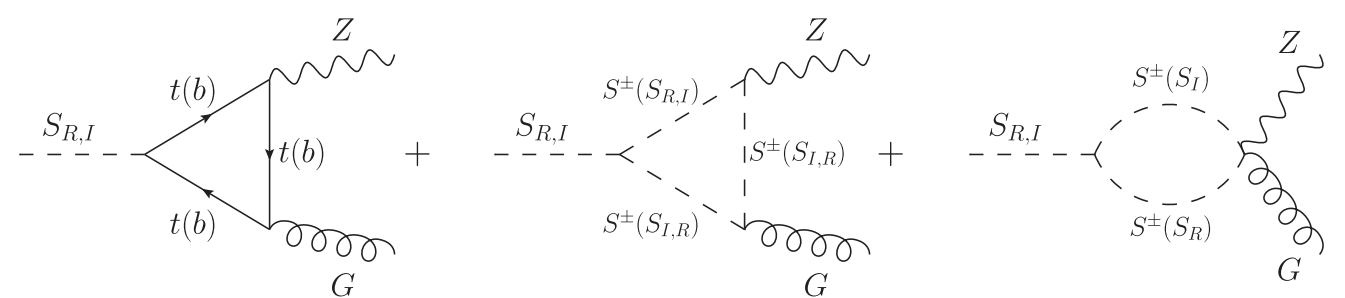

FIG. 3. One-loop diagrams contributing to the factors appearing in Eq. (4) for the $S_{R, I} Z g$ coupling.

$$
\begin{aligned}
& \tilde{F}_{R}^{\gamma g}=\left[-\eta_{U} s_{U} \frac{m_{t}^{2}}{m_{R}^{2}} f\left(\frac{m_{t}^{2}}{m_{R}^{2}}\right)+\eta_{D} s_{D} \frac{1}{2} \frac{m_{b}^{2}}{m_{R}^{2}} f\left(\frac{m_{b}^{2}}{m_{R}^{2}}\right)\right] \\
& \tilde{F}_{I}^{\gamma g}=\left[-\eta_{U} c_{U} \frac{m_{t}^{2}}{m_{I}^{2}} f\left(\frac{m_{t}^{2}}{m_{I}^{2}}\right)-\eta_{D} c_{D} \frac{1}{2} \frac{m_{b}^{2}}{m_{I}^{2}} f\left(\frac{m_{b}^{2}}{m_{I}^{2}}\right)\right] .
\end{aligned}
$$

We note that the scalar loop contribution to $S_{R} \rightarrow \gamma g$ vanishes in the custodial symmetry limit. In the custodial and $C P$ symmetry limits, the scalar loops do not affect these processes.

\section{C. $S_{R, I} \rightarrow Z g$}

In this case, the relevant diagrams are shown in Fig. 3 and result in form factors given by

$$
\begin{aligned}
F_{R}^{Z g}= & \left\{-\eta_{U} c_{U} \frac{\left(3 c_{W}^{2}-5 s_{W}^{2}\right)}{s_{2 W}}\right. \\
& \times\left[I_{1}\left(\frac{m_{t}^{2}}{m_{R}^{2}}, \frac{m_{t}^{2}}{m_{Z}^{2}}\right)-I_{2}\left(\frac{m_{t}^{2}}{m_{R}^{2}}, \frac{m_{t}^{2}}{m_{Z}^{2}}\right)\right] \\
& +\eta_{D} c_{D} \frac{\left(3 c_{W}^{2}-s_{W}^{2}\right)}{s_{2 W}}\left[I_{1}\left(\frac{m_{b}^{2}}{m_{R}^{2}}, \frac{m_{b}^{2}}{m_{Z}^{2}}\right)-I_{2}\left(\frac{m_{b}^{2}}{m_{R}^{2}}, \frac{m_{b}^{2}}{m_{Z}^{2}}\right)\right] \\
& \left.-\frac{9}{4} \frac{v^{2}}{m_{S^{ \pm}}^{2}} \frac{1}{t_{2 W}}\left(\lambda_{4} c_{4}-\lambda_{5} c_{5}\right)\left[I_{1}\left(\frac{m_{S^{ \pm}}^{2}}{m_{R}^{2}}, \frac{m_{S^{ \pm}}^{2}}{m_{Z}^{2}}\right)\right]\right\} \\
F_{I}^{Z g}= & \left\{\eta_{U} s_{U} \frac{\left(3 c_{W}^{2}-5 s_{W}^{2}\right)}{s_{2 W}}\left[I_{1}\left(\frac{m_{t}^{2}}{m_{I}^{2}}, \frac{m_{t}^{2}}{m_{Z}^{2}}\right)-I_{2}\left(\frac{m_{t}^{2}}{m_{I}^{2}}, \frac{m_{t}^{2}}{m_{Z}^{2}}\right)\right]\right. \\
& +\eta_{D} s_{D} \frac{\left(3 c_{W}^{2}-s_{W}^{2}\right)}{s_{2 W}}\left[I_{1}\left(\frac{m_{b}^{2}}{m_{I}^{2}}, \frac{m_{b}^{2}}{m_{Z}^{2}}\right)-I_{2}\left(\frac{m_{b}^{2}}{m_{I}^{2}}, \frac{m_{b}^{2}}{m_{Z}^{2}}\right)\right] \\
& \left.+\frac{9}{4} \frac{v^{2}}{m_{S^{ \pm}}^{2}} \frac{1}{t_{2 W}}\left(\lambda_{4} s_{4}-\lambda_{5} s_{5}\right)\left[I_{1}\left(\frac{m_{S^{ \pm}}^{2}}{m_{I}^{2}}, \frac{m_{S^{ \pm}}^{2}}{m_{Z}^{2}}\right)\right]\right\}
\end{aligned}
$$

$$
\begin{aligned}
\tilde{F}_{R}^{Z g}= & \frac{1}{\left(m_{R}^{2}-m_{Z}^{2}\right)} \\
& \times\left\{-\eta_{U} s_{U} \frac{\left(3 c_{W}^{2}-5 s_{W}^{2}\right)}{s_{2 W}} m_{t}^{2}\left[f\left(\frac{m_{t}^{2}}{m_{R}^{2}}\right)-f\left(\frac{m_{t}^{2}}{m_{Z}^{2}}\right)\right]\right. \\
& \left.+\eta_{D} s_{D} \frac{\left(3 c_{W}^{2}-s_{W}^{2}\right)}{s_{2 W}} m_{b}^{2}\left[f\left(\frac{m_{b}^{2}}{m_{R}^{2}}\right)-f\left(\frac{m_{b}^{2}}{m_{Z}^{2}}\right)\right]\right\}
\end{aligned}
$$

$$
\begin{aligned}
\tilde{F}_{I}^{Z g}= & \frac{1}{\left(m_{I}^{2}-m_{Z}^{2}\right)} \\
& \times\left\{-\eta_{U} c_{U} \frac{\left(3 c_{W}^{2}-5 s_{W}^{2}\right)}{s_{2 W}} m_{t}^{2}\left[f\left(\frac{m_{t}^{2}}{m_{I}^{2}}\right)-f\left(\frac{m_{t}^{2}}{m_{Z}^{2}}\right)\right]\right. \\
& \left.-\eta_{D} c_{D} \frac{\left(3 c_{W}^{2}-s_{W}^{2}\right)}{s_{2 W}} m_{b}^{2}\left[f\left(\frac{m_{b}^{2}}{m_{I}^{2}}\right)-f\left(\frac{m_{b}^{2}}{m_{Z}^{2}}\right)\right]\right\} .
\end{aligned}
$$

The functions $I_{1,2}(x, y)$ already appear in the scalar contributions to $H \rightarrow Z \gamma$ [38] and are given below. Once again, the scalar loop contributions to $S_{R} \rightarrow Z g$ vanish if custodial symmetry is imposed, and those to $S_{I} \rightarrow Z g$ vanish when $C P$ symmetry is imposed.

$$
\text { D. } S^{+} \rightarrow W^{+} g
$$

Finally, the diagrams for $S^{+} \rightarrow W^{+} g$ are shown in Fig. 4. The complete result is rather cumbersome and quite complicated; therefore to simplify the calculation we preferred to choose the case where $m_{S^{ \pm}}=m_{I}$, and we present it in the Appendix. It simplifies considerably if $m_{b} \rightarrow 0$, and we treat the scalars as degenerate. In this case, and separating the quark and scalar loop contributions into $F^{W g}=F_{q}^{W g}+F_{S}^{W g}$ we find

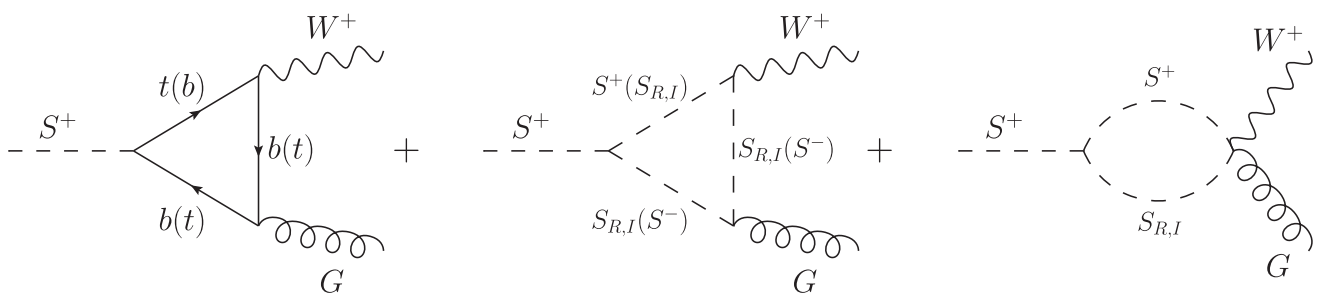

FIG. 4. One-loop diagrams contributing to the factors appearing in Eq. (4) for the $S^{ \pm} W^{\mp} g$ coupling. 
(i) the quark loop contributions in the limit $m_{b} \rightarrow 0$ become

$$
\begin{aligned}
F_{q}^{W g}= & \frac{3 \eta_{U} e^{i \alpha_{U}}\left|V_{t b}\right|^{2} m_{t}^{2}}{2 m_{I}^{2}\left(m_{I}^{2}-m_{W}^{2}\right)^{2} s_{W}}\left[\frac{1}{2} m_{I}^{2}\left(m_{I}^{2}-2 m_{t}^{2}-m_{W}^{2}\right) .\right. \\
& \left(2 \mathrm{Li}_{2}\left(\frac{m_{t}^{2}}{m_{I}^{2}}\right)-2 \mathrm{Li}_{2}\left(\frac{m_{t}^{2}}{m_{W}^{2}}\right)+\log \left(\frac{m_{W}^{2}}{m_{I}^{2}}\right) \log \left(\frac{m_{t}^{4}}{m_{I}^{2} m_{W}^{2}}\right)\right)-2 m_{I}^{2}\left(m_{I}^{2}-m_{W}^{2}\right) \\
& \left.+2 m_{I}^{2}\left(m_{t}^{2}-m_{W}^{2}\right) \log \left(\frac{m_{t}^{2}-m_{W}^{2}}{m_{t}^{2}}\right)+2 m_{W}^{2}\left(m_{I}^{2}-m_{t}^{2}\right) \log \left(\frac{m_{t}^{2}-m_{I}^{2}}{m_{t}^{2}}\right)\right],
\end{aligned}
$$

(ii) the scalar loop contributions when all scalars are degenerate and $m_{W} \ll m_{S}^{ \pm}$become

$$
F_{S}^{W g}=\frac{9 v^{2}}{4 m_{S}^{2} s_{W}} I_{s}(1)\left(\lambda_{4}-\lambda_{5}\right)\left(1+\mathcal{O}\left(\frac{m_{W}^{2}}{m_{S}^{2}}\right)\right)
$$

In the same limit,

$$
\tilde{F}^{W g}=\frac{1}{s_{W}}\left\{-\frac{3 i \eta_{U}\left|V_{t b}\right|^{2} m_{t}^{2}}{4\left(m_{I}^{2}-m_{W}^{2}\right)}\left(2 \mathrm{Li}_{2}\left(\frac{m_{t}^{2}}{m_{I}^{2}}\right)-2 \mathrm{Li}_{2}\left(\frac{m_{t}^{2}}{m_{W}^{2}}\right)+\log \left(\frac{m_{W}^{2}}{m_{I}^{2}}\right) \log \left(\frac{m_{t}^{4}}{m_{I}^{2} m_{W}^{2}}\right)\right)\right\} .
$$

\section{E. Loop functions}

The functions appearing in the above results are given by

$$
\begin{aligned}
I_{q}(x)= & 2 x+x(4 x-1) f(x), \\
I_{s}(x)= & -(1+2 x f(x)), \\
I_{1}(x, y)= & \frac{2 x y}{(x-y)}-\frac{4 x^{2} y^{2}}{(x-y)^{2}}[f(x)-f(y)] \\
& +\frac{4 x^{2} y}{(x-y)^{2}}[g(x)-g(y)] \\
I_{2}(x, y)= & \frac{x y}{(x-y)}[f(x)-f(y)],
\end{aligned}
$$

where

$$
f(x)= \begin{cases}\frac{1}{2}\left(\ln \left(\frac{1+\sqrt{1-4 x}}{1-\sqrt{1-4 x}}\right)-i \pi\right)^{2} & \text { for } x<\frac{1}{4} \\ -2\left(\arcsin \left(\frac{1}{2 \sqrt{x}}\right)\right)^{2} & \text { for } x>\frac{1}{4}\end{cases}
$$

and

$g(x)= \begin{cases}\frac{1}{2} \sqrt{1-4 x}\left(\ln \left(\frac{1+\sqrt{1-4 x}}{1-\sqrt{1-4 x}}\right)-i \pi\right) & \text { for } x<\frac{1}{4} \\ \sqrt{4 x-1} \arcsin \left(\frac{1}{2 \sqrt{x}}\right) & \text { for } x>\frac{1}{4}\end{cases}$

The special value appearing for degenerate scalar masses, $I_{S}(1)=\frac{\pi^{2}}{9}-1$.

\section{DECAY WIDTHS IN DIFFERENT SCENARIOS}

The decay widths are given in terms of the couplings defined in Eq. (4) by

$$
\begin{aligned}
\Gamma\left(S_{R, I} \rightarrow g g\right)= & \frac{5}{12 \pi}\left(\frac{\alpha_{s}}{8 \pi v}\right)^{2} m_{R, I}^{3}\left(\left|F_{R, I}^{g g}\right|^{2}+\left|\tilde{F}_{R, I}^{g g}\right|^{2}\right) \\
\Gamma\left(S_{R, I} \rightarrow \gamma g\right)= & \frac{1}{8 \pi}\left(\frac{\alpha \alpha_{s}}{(3 \pi v)^{2}}\right) m_{R, I}^{3}\left(\left|F_{R, I}^{\gamma g}\right|^{2}+\left|\tilde{F}_{R, I}^{\gamma g}\right|^{2}\right) \\
\Gamma\left(S_{R, I} \rightarrow Z g\right)= & \frac{1}{8 \pi}\left(\frac{\alpha \alpha_{s}}{(12 \pi v)^{2}}\right) \frac{\left(m_{R, I}^{2}-m_{Z}^{2}\right)^{3}}{m_{R, I}^{3}} \\
& \times\left(\left|F_{R, I}^{Z g}\right|^{2}+\left|\tilde{F}_{R, I}^{Z g}\right|^{2}\right) \\
\Gamma\left(S^{ \pm} \rightarrow W^{ \pm} g\right)= & \frac{1}{8 \pi}\left(\frac{\alpha \alpha_{s}}{(12 \pi v)^{2}}\right) \frac{\left(m_{S^{+}}^{2}-m_{W}^{2}\right)^{3}}{m_{S^{+}}^{3}} \\
& \times\left(\left|F_{R, I}^{W g}\right|^{2}+\left|\tilde{F}_{R, I}^{W g}\right|^{2}\right) .
\end{aligned}
$$

Numerically, these result in small branching ratios that are negligible for phenomenology except in special cases.

\section{A. General remarks on parameter space}

There are two cases in which the $V g$ modes are important, and they both rely on mechanisms to suppress scalar decay into heavy quarks.

(i) The $S_{R, I}$ neutral resonances will decay predominantly into top pairs, and $S^{ \pm}$will decay predominantly into top-bottom pairs if those channels are kinematically available. This means that the loop induced modes become important for the mass ranges, 


$$
\begin{aligned}
& 100 \mathrm{GeV} \lesssim m_{R, I} \lesssim 350 \mathrm{GeV}, \\
& 100 \mathrm{GeV} \lesssim m_{S^{ \pm}} \lesssim 175 \mathrm{GeV} .
\end{aligned}
$$

The lower limit corresponds approximately to the exclusion set by the large electron positron collider for scalar pair production [9]. Scalar decay into two jets through couplings to the lighter quarks are not suppressed in these ranges and in some instances will dominate as shown below.

(ii) The decays to $t \bar{t}$ or $t \bar{b}$ can also be suppressed with very small values of $\eta_{U}$. This would also suppress the production mechanism for a single scalar but would not affect the cross section for pair-production which depends only on the QCD coupling constant, and at $13 \mathrm{TeV}$ is approximately $0.2 \mathrm{pb}$. [8,22]. Reducing $\eta_{U}$ also reduces the top-quark contribution to the loop decays, which is dominant in most cases.

(iii) When $\eta_{U}$ is small, $S_{R, I}$ will also decay predominantly into bottom pairs unless $\eta_{D}$ is also small. It is possible for the color octet scalars to have very small Yukawa couplings or to introduce discrete symmetries [39] or additional scalar multiplets [16] so that they vanish, resulting in fermiophobic scenarios [40].

(iv) Large values of $\lambda_{4,5}$, that obey the condition $\lambda_{4}=\lambda_{5}^{\star}$, can significantly affect the $g g$ channel but not the other ones.

(v) It is possible to enhance the $\gamma g$ or $Z g$ modes relative to the $g g$ mode if the sign of $\lambda_{4}$ results in destructive interference with the fermion loops for $g g$.

(vi) Kinematic windows can also be used to suppress decays between the different scalars. For example, choosing $\lambda_{2}=0$ results in degeneracy between $S_{I}$ and $S_{R}$ thus preventing (on shell) decays between them.

\section{B. Scalar potential with $\lambda_{5} \neq \lambda_{4}^{\star}$}

One of the conditions arising from imposing custodial symmetry is $\lambda_{4}=\lambda_{5}^{\star}$. These parameters, however, do not affect the $W, Z$ masses until at least the two-loop level so

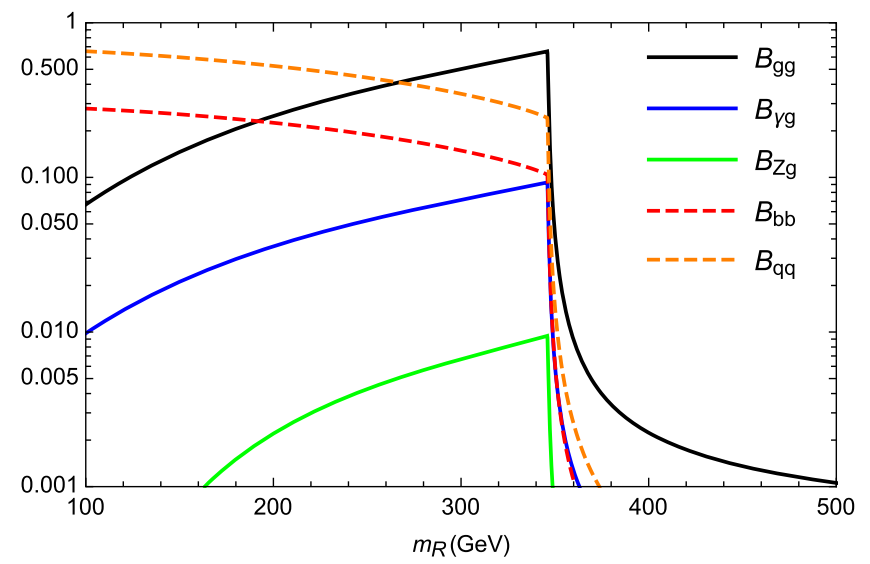

constraints from the $\rho$ parameter are much weaker than those on $\lambda_{2,3}$. Entertaining this possibility permits a suppression of multijet modes (via the $S \rightarrow g g$ decay) in favor of the $\gamma g$ and $\mathrm{Zg}$ modes. We illustrate this for two cases,

(i) $C P$ conserving scenario with $\lambda_{5}=-\lambda_{4}$. This completely removes the scalar loop contributions in the $S_{R, I} \rightarrow g g$ modes while enhancing them in $S_{R, I} \rightarrow$ $\gamma g, Z g$ as well as in $S^{ \pm} \rightarrow W^{ \pm} g$.

(ii) $C P$ violating scenario with $\left|\lambda_{5}\right|=\left|\lambda_{4}\right|$, but both have a phase of $\pi / 2$. This also removes the scalar loop contributions in the $S_{R, I} \rightarrow g g$ and enhances them in $S_{R, I} \rightarrow \gamma g, Z g$; this time via $C P$ violating contributions.

\section{Parameter choices}

For our numerical results we will keep $\eta_{U} \leq 5$, below its unitarity constraint [15]. We will choose a smaller $\eta_{D}$, typically $\eta_{D} \leq 1$ as we do not want to enhance the $b \bar{b}$ decay modes. To illustrate the fermiophobic scenario we set $\eta_{U}=0$ but keep $\eta_{D}$ small to show the interplay between the different modes. In all cases we keep $\left|\lambda_{4,5}\right| \leq 10$, which is below its tree level unitarity constraint [15] and within the range of next to leading order unitarity constraints [41]. We select a few benchmarks for nonzero $C P$ phases in the scalar potential.

\section{NUMERICAL RESULTS}

\section{A. Low scalar mass window}

When the Yukawa couplings are of order one, the decay modes into third generation fermions are completely dominant. In this case the loop induced $V g$ modes only become important for sufficiently low scalar masses. To illustrate this scenario we select $\eta_{U}=5, \eta_{D}=1$ in Figs. 5 and 6 where the $V g$ loop modes are shown as solid lines and tree-level $q \bar{q}$ modes as dashed lines. For masses $m_{R}$ above $2 m_{t}$ the $\mathcal{B}\left(S_{R} \rightarrow t \bar{t}\right) \approx 1$ unless $\eta_{U} \lesssim \eta_{D} m_{b} / m_{t}$, in which

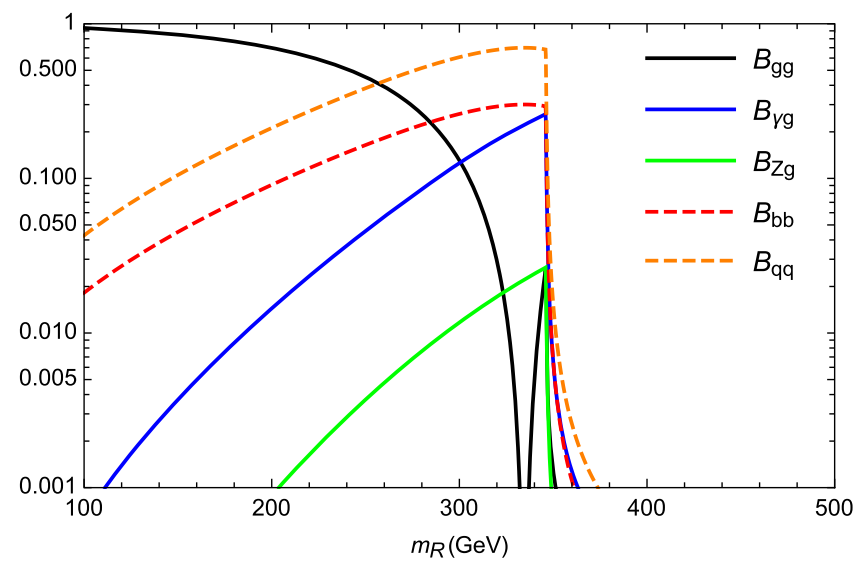

FIG. 5. Branching ratios for $S_{R}$ decay in the fermiophilic scenario $\eta_{U}=5, \eta_{D}=1$ (left panel: $\lambda_{4,5}=0$, right panel: $\lambda_{4}=\lambda_{5}=-10$. Above the $t \bar{t}$ threshold, $\mathcal{B}\left(S_{R} \rightarrow t \bar{t}\right)$ rises rapidly to $100 \%$. 

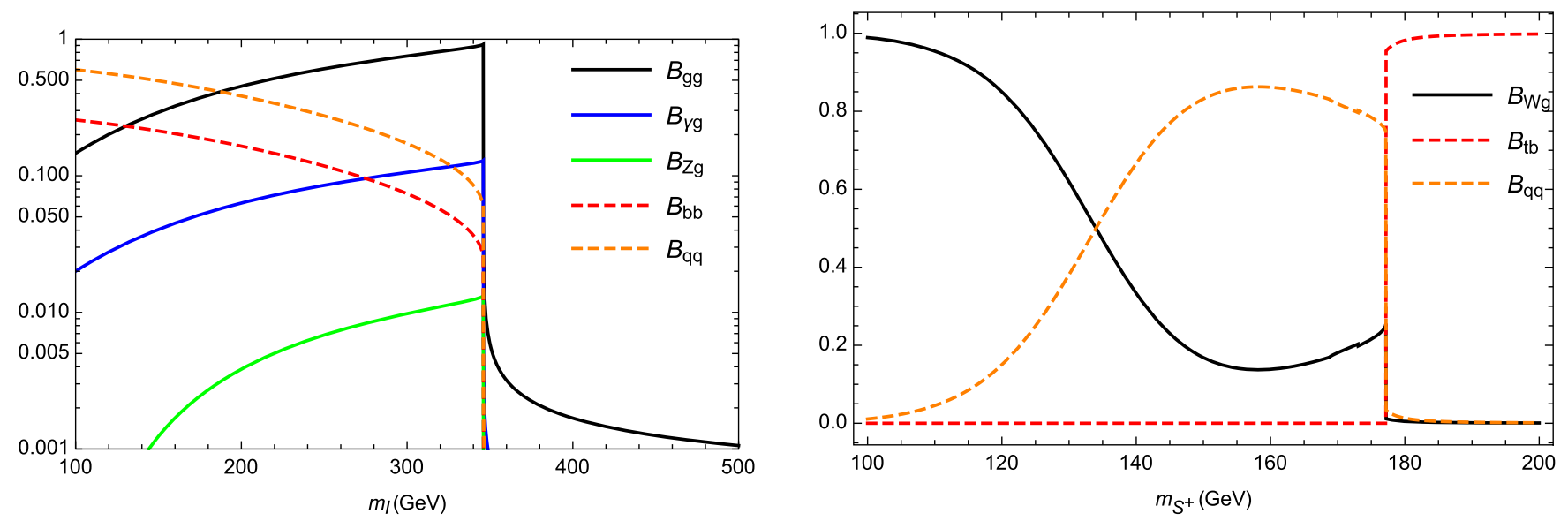

FIG. 6. Branching ratios for $S_{I}$ left panel ( $S^{ \pm}$right panel) decay for parameter values $\eta_{U}=5$ and $\eta_{D}=1$. Above the $t \bar{t}$ threshold, $\mathcal{B}\left(S_{I} \rightarrow t \bar{t}\right)$ rises rapidly to $100 \%$.

case $\mathcal{B}\left(S_{R} \rightarrow b \bar{b}\right)$ becomes comparable. The choice $\eta_{U}=5$ for $m_{R}$ below $2 m_{t}$ enhances the rate into $V g$ modes relative to tree-level $b \bar{b}$ modes through the top-quark loop contribution.

The relevant branching ratios for $S_{R}$ decay are shown in Fig. 5. The figure illustrates the interplay between $g g, q q$ (light quarks) and $b \bar{b}$ modes which are the most important ones in this case. On the left panel we set $\lambda_{4,5}=0$, so the contributions to $V g$ modes are only from quark loops. For the right panel we used $\lambda_{4}=\lambda_{5}=-10$ in order to enhance the contribution of the scalar loops, and with the sign chosen to suppress the $g g$ mode near the $t \bar{t}$ threshold. In this case the $\gamma g$ mode can reach branching ratios near $12 \%$ and the $\mathrm{Zg}$ mode near $1 \%$.

The corresponding decay modes for $S_{I}$ are shown in the left panel of Fig. 6. As mentioned before, $\lambda_{4,5}$ do not affect $S_{I} \rightarrow g g$ if custodial symmetry is imposed $\left(\lambda_{5}=\lambda_{4}^{\star}\right)$, and they do not affect $S_{I} \rightarrow(\gamma / Z) g$ if $C P$ symmetry is imposed. The branching ratios shown in Fig. 6 will thus vary only with the ratio $\eta_{U} / \eta_{D}$. The right panel of the same figure illustrates decay modes of the charged scalar, for which $\mathrm{Wg}$ mode can dominate for values of $m_{S^{ \pm}}$below the $t \bar{b}$ threshold. The $q q$ light-quark mode below $t \bar{b}$ threshold is dominated by $c \bar{b}$ quarks.

\section{B. Fermiophobic scenario}

In the limit of vanishing Yukawa couplings, $\eta_{U, D}=0$, the tree-level decays of $S$ into fermion pairs vanish, and the one-loop modes are driven by scalar loop contributions. For

TABLE I. Sample parameter points with $\eta_{U, D}=0$.

\begin{tabular}{lccccc}
\hline \hline$\phi_{4}=\phi_{5}=0$ & $\lambda_{5}=\lambda_{4}$ & $\lambda_{5}=-\lambda_{4}$ & $\phi_{4}=\phi_{5}=\frac{\pi}{2}$ & $\lambda_{5}=\lambda_{4}$ & $\lambda_{5}=-\lambda_{4}$ \\
\hline $\mathcal{B}\left(S_{R} \rightarrow g g\right)$ & 1 & 0 & $\mathcal{B}\left(S_{I} \rightarrow g g\right)$ & 1 & 0 \\
$\mathcal{B}\left(S_{R} \rightarrow \gamma g\right)$ & 0 & $71.5 \%$ & $\mathcal{B}\left(S_{I} \rightarrow \gamma g\right)$ & 0 & $71.5 \%$ \\
$\mathcal{B}\left(S_{R} \rightarrow Z g\right)$ & 0 & $28.5 \%$ & $\mathcal{B}\left(S_{I} \rightarrow Z g\right)$ & 0 & $28.5 \%$ \\
\hline \hline
\end{tabular}

the charged scalar we have in this case $\mathcal{B}\left(S^{ \pm} \rightarrow W^{ \pm} g\right) \approx 1$ provided $\lambda_{5} \neq \lambda_{4}$. The situation for the neutral scalars is more complicated, and we show some limiting cases in Table I. For small, but nonzero $\eta_{D}$, decays into $b$ quarks compete with the loop-induced modes. We illustrate this interplay for selected parameter points in Fig. 7, where $\eta_{U}=0$ in all cases. The top two panels show $S_{R}$ branching ratios: on the left we take $\lambda_{4}=\lambda_{5}$ with no phases, which removes the $\gamma g$ and $Z g$ modes. The ratio $\Gamma\left(S_{R} \rightarrow g g\right) /$ $\Gamma\left(S_{R} \rightarrow b b\right)$ increases with increasing $\lambda_{4} / \eta_{D}$; on the right we still take $\lambda_{4}=\lambda_{5}$ but allow a $90^{\circ}$ phase which removes the $g g$ mode in favor of $\gamma g$ and $Z g$. The two panels in the center row illustrate the dependence on $m_{R}$ and $\eta_{D}$ for certain fixed choices of $\lambda_{4,5}$, again for $S_{R}$ decay. Finally in the bottom row, we show branching ratios for $S_{I}$ decay on the left panel and for $S^{ \pm}$decay on the right panel. In both cases we illustrate the dependence on the scalar mass for fixed choices of $\eta_{D}$ and $\lambda_{4,5}$. The message from Fig. 7 is that the $V g$ modes can be dominant in fermiophobic scenarios, and that their relative importance varies across the parameter space. The study of $V g$ modes is therefore necessary to fully constrain models with new colored scalars.

\section{Comparison with the literature}

A recent analysis of $g g, \gamma g$ and $Z g$ modes at LHC appeared in [29]. This study is quite different from ours, as it concerns models where the effective vertices arise through a Wess-Zumino term in composite models. They offer a parametrization of the effective vertices that we can use to compare to our results, they write

$$
\begin{aligned}
\mathcal{L}_{\Phi} \supset & i C_{t} \frac{m_{t}}{f_{\Phi}} \Phi^{a} \bar{t} \gamma_{5} \frac{\lambda^{a}}{2} t+\frac{\alpha_{s} \kappa_{g}}{8 \pi f_{\Phi}} \Phi^{a} \epsilon^{\mu \nu \rho \sigma} \\
\times & \left.\times \frac{1}{2} d^{a b c} G_{\mu \nu}^{b} G_{\rho \sigma}^{c}+\frac{e \kappa_{\gamma}}{g_{s} \kappa_{g}} G_{\mu \nu}^{a} F_{\rho \sigma}-\frac{e \tan _{W} \kappa_{Z}}{g_{s} \kappa_{g}} G_{\mu \nu}^{a} Z_{\rho \sigma}\right],
\end{aligned}
$$



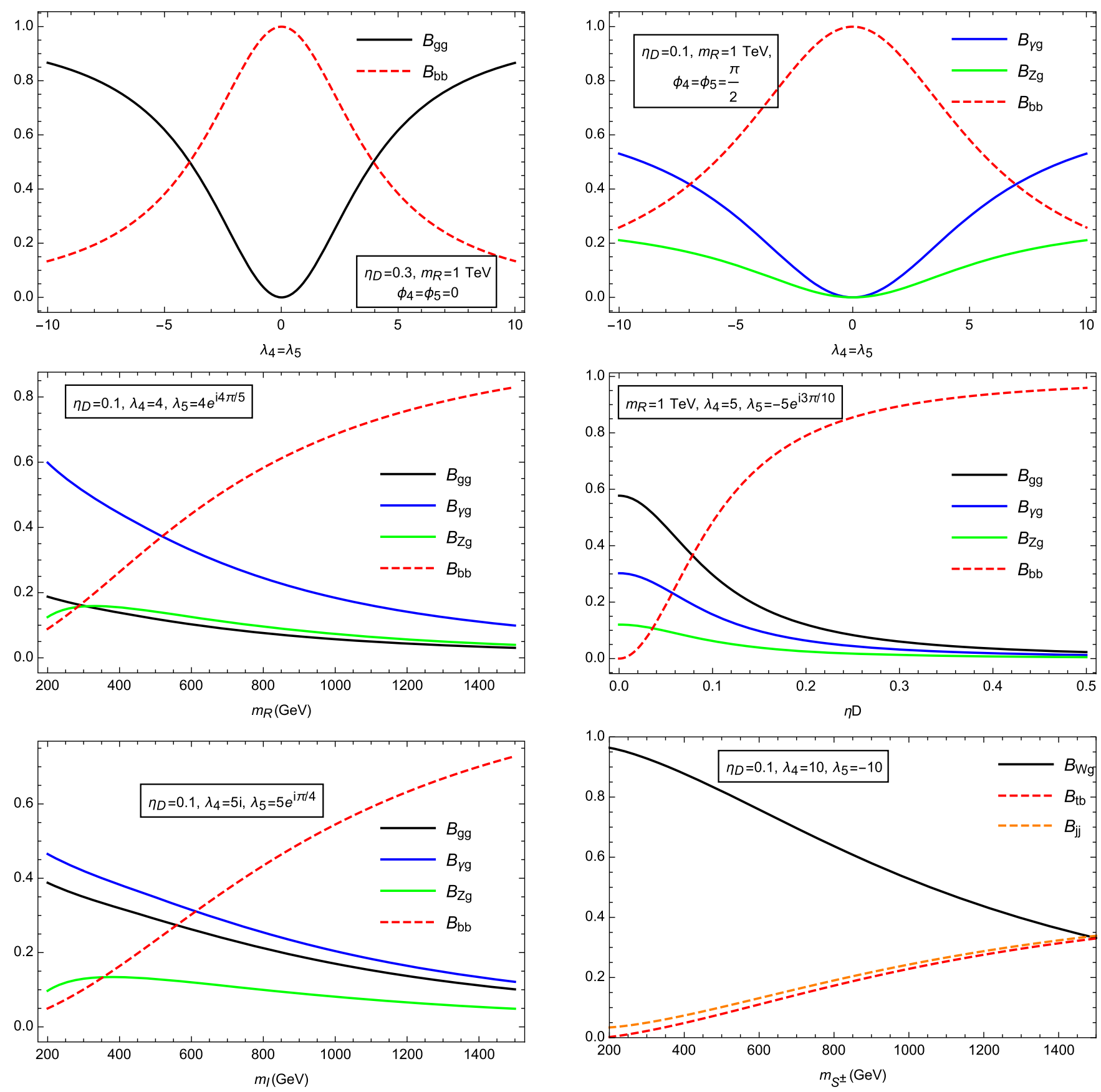

FIG. 7. Illustrative parameter points for $S_{R}$ decay modes, top four panels; $S_{I}$ decay modes, bottom left; and $S^{ \pm}$decay modes, bottom right. In all cases $\eta_{U}=0$.

where $\Phi$ is assumed to be a composite octet pseudoscalar with mass scale $f_{\Phi}$. Our results are given in the form of Eq. (4), indicating that the MW model at one-loop produces the effective couplings in Eq. (25) as

$C_{t}=\frac{f_{\Phi}}{v} \eta_{U}, \quad \kappa_{g}=\frac{f_{\Phi}}{v} \tilde{F}_{I}^{g g}, \quad \kappa_{\gamma}=\frac{8}{3} \frac{f_{\Phi}}{v} \tilde{F}_{I}^{\gamma g}$,

$\kappa_{Z}=\frac{2}{3} \cot _{W} \frac{f_{\Phi}}{v} \tilde{F}_{I}^{Z g}$ if we identify the neutral pseudoscalar $S_{I}$ with the composite $\Phi$. We find, in agreement with [29], that the loop-induced modes are phenomenologically relevant when $C_{t} \ll \kappa_{g}$, which in the MW model corresponds to a dominance of the scalar loops due to vanishing (or very small) $\eta_{U}$.

In addition, the results of [29] are presented in terms of the ratio $\kappa_{\gamma} / \kappa_{g}$. In the MW model, this ratio can be tuned from 0 to 1 as can be seen in Table I. In the regime where 


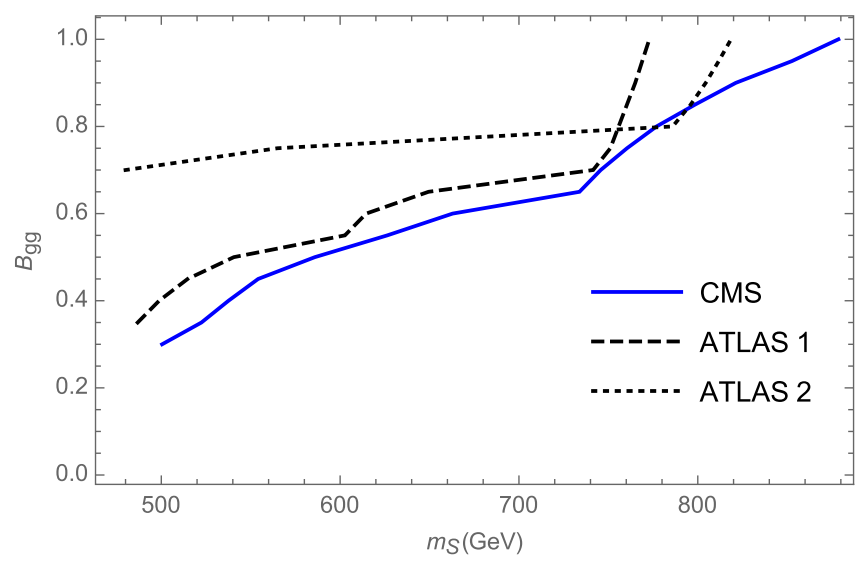

FIG. 8. Limits on $\mathcal{B}(S \rightarrow g g)$ from ATLAS [43] and CMS [42] searches for pair-produced resonances decaying to four jets as discussed in the text.

the $\gamma g$ and $Z g$ modes are large, single production of $S$ at the LHC is highly suppressed. Any bounds would come from QCD pair production of $S S$ followed by decays into $\gamma / Z j$.

The closest experimental studies we can find consist of pair produced resonances decaying to four jets without b-tags. These studies are applicable to $S S$ pair production followed by four jet decays and was considered by us in [22]. These constraints have been updated by two studies: a CMS study of pair-produced squarks decaying to light quark pairs [42] with $35.9 \mathrm{fb}^{-1}$ at $13 \mathrm{TeV}$; and an ATLAS study of pair produced resonances (squarks and colorons) in four jet final states [43] with $36.7 \mathrm{fb}^{-1}$ at $13 \mathrm{TeV}$. Following our procedure in [22] we can extract a limit on $\mathcal{B}(S \rightarrow g g)$ shown in Fig. 8. The limits are obtained from the $95 \% \mathrm{cl}$ observed upper limit in $\sigma(p p \rightarrow \tilde{t} \overline{\tilde{t}})$ in Fig. 11 of [42] (labeled CMS in our figure) and from the corresponding limits on $\sigma(p p \rightarrow \tilde{t} \tilde{\tilde{t}})$ [Fig. 9(a)] and $\sigma(p p \rightarrow \rho \rho)$ [ $\rho$ are colorons, Fig. 9(c)] of [43] (labeled ATLAS 1 and ATLAS 2 in our figure). These results are encouraging for future studies at LHC as they suggest that the two gluon decay mode could be constrained from the four-jet channel. The key issue in constraining the $V j$ modes will then be the ability of ATLAS and CMS to reconstruct these final states. The theoretical study in [29] estimates the relevant backgrounds and finds regions in the $\left[m_{\Phi}, \mathcal{B}(\Phi \rightarrow g \gamma)\right]$ plane that can be covered by $j j j \gamma$ and

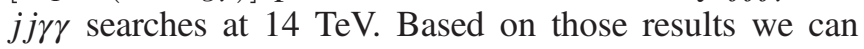
conclude that these modes can also be used to constrain the MW model in the future.

\section{SUMMARY AND CONCLUSION}

We have presented for the first time explicit one-loop results for the decay modes of a scalar color octet $S^{0} \rightarrow \gamma g$, $S^{0} \rightarrow Z g$ and $S^{ \pm} \rightarrow W^{ \pm} g$ in the MW model. These results arise from heavy quark loops proportional to the Yukawa couplings of the colored scalars and from colored scalar loops proportional to the triple scalar coupling in the potential. We have further identified the regions of parameter space where these modes become important. These regions correspond to fermiophobic scenarios and/or to low scalar masses for which decays into heavy quarks are kinematically forbidden.

\section{ACKNOWLEDGMENTS}

We thank Thomas Flacke for pointing out these vertices did not appear in the literature. We also thank Vladyslav Shtabovenko for very useful discussions about the automation of loop calculations with the FeynCalc package.

\section{APPENDIX A: COMPLETE LOOP FACTORS FOR $S^{ \pm} \rightarrow W^{ \pm} g$}

The complete expressions we obtain in this case can be written in terms of the Passarino-Veltman function $C_{0}$ as follows:

$$
\begin{aligned}
F^{W g}= & \frac{1}{\left(m_{I}^{2}-m_{W}^{2}\right)^{2} s_{W}}\left\{3 | V _ { t b } | ^ { 2 } \left[\left(m_{t}^{2} \eta_{U} e^{i \alpha_{U}}-m_{b}^{2} \eta_{D} e^{-i \alpha_{D}}\right)\right.\right. \\
& \times\left(\left(m_{W}^{2}-m_{b}^{2}-m_{t}^{2}\right) I^{+}\left(\frac{m_{b}^{2} m_{t}^{2}}{\left(m_{W}^{2}-m_{b}^{2}-m_{t}^{2}\right)^{2}}\right)-\frac{m_{W}^{2}}{m_{I}^{2}}\left(m_{I}^{2}-m_{b}^{2}-m_{t}^{2}\right) I^{-}\left(\frac{m_{b}^{2} m_{t}^{2}}{\left(m_{I}^{2}-m_{b}^{2}-m_{t}^{2}\right)^{2}}\right)\right) \\
& -\frac{m_{b}^{2}}{2}\left(m_{I}^{2}-m_{W}^{2}\right)\left(2 m_{t}^{2} \eta_{U} e^{i \alpha_{U}}+\left(m_{I}^{2}-2 m_{b}^{2}-m_{W}^{2}\right) \eta_{D} e^{-i \alpha_{D}}\right) C_{0}\left(0, m_{I}^{2}, m_{W}^{2}, m_{b}^{2}, m_{b}^{2}, m_{t}^{2}\right) \\
& +\frac{m_{t}^{2}}{2}\left(m_{I}^{2}-m_{W}^{2}\right)\left(\left(m_{I}^{2}-2 m_{t}^{2}-m_{W}^{2}\right) \eta_{U} e^{i \alpha_{U}}+2 m_{b}^{2} \eta_{D} e^{-i \alpha_{D}}\right) C_{0}\left(0, m_{I}^{2}, m_{W}^{2}, m_{t}^{2}, m_{t}^{2}, m_{b}^{2}\right) \\
& \left.-\left(m_{t}^{2} \eta_{U} e^{i \alpha_{U}}-m_{b}^{2} \eta_{D} e^{-i \alpha_{D}}\right)\left(m_{I}^{2}-m_{W}^{2}\right)\left(1-\frac{\left(m_{t}^{2}-m_{b}^{2}\right)}{2 m_{I}^{2}} \log \left(\frac{m_{b}^{2}}{m_{t}^{2}}\right)\right)\right] \\
& +\frac{9}{4} v^{2}\left[i\left(\lambda_{4} s_{4}-\lambda_{5} s_{5}\right)\left(-2 m_{W}^{2} g\left(\frac{m_{I}^{2}}{m_{W}^{2}}\right)+2 m_{I}^{2} f\left(\frac{m_{I}^{2}}{m_{W}^{2}}\right)+m_{I}^{2} I_{s}(1)+m_{W}^{2}(1+2 g(1))\right)\right.
\end{aligned}
$$




$$
\begin{aligned}
& +\left(\lambda_{4} c_{4}-\lambda_{5} c_{5}\right)\left(m_{W}^{2}-m_{R}^{2}-m_{I}^{2}\right) I^{+}\left(\frac{m_{R}^{2} m_{I}^{2}}{\left(m_{W}^{2}-m_{R}^{2}-m_{I}^{2}\right)^{2}}\right) \\
& -\left(\lambda_{4} c_{4}-\lambda_{5} c_{5}\right)\left(-\frac{m_{W}^{2} m_{R}^{2}}{m_{I}^{2}} I^{+}\left(\frac{m_{I}^{2}}{m_{R}^{2}}\right)+\frac{\left(m_{I}^{2}-m_{W}^{2}\right)}{2} \frac{\left(m_{I}^{2}-m_{R}^{2}\right)}{m_{I}^{2}} \log \left(\frac{m_{I}^{2}}{m_{R}^{2}}\right)\right) \\
& -\left(m_{I}^{2}-m_{W}^{2}\right)\left(\lambda_{4} c_{4}-\lambda_{5} c_{5}\right) \\
& \times\left(m_{R}^{2} C_{0}\left(0, m_{I}^{2}, m_{W}^{2}, m_{R}^{2}, m_{R}^{2}, m_{I}^{2}\right)+m_{I}^{2} C_{0}\left(0, m_{I}^{2}, m_{W}^{2}, m_{I}^{2}, m_{I}^{2}, m_{R}^{2}\right)\right) \\
& \left.\left.-\left(\lambda_{4} c_{4}-\lambda_{5} c_{5}\right)\left(m_{I}^{2}-m_{W}^{2}\right)\right]\right\} .
\end{aligned}
$$

The remaining loop functions can be written as

$$
\begin{gathered}
I^{+}(x)= \begin{cases}\sqrt{1-4 x} \ln \left(\frac{1-\sqrt{1-4 x}}{2 \sqrt{x}}\right) & \text { for } x<\frac{1}{4} \\
\sqrt{4 x-1} \arcsin \left(\frac{\sqrt{4 x-1}}{2 \sqrt{x}}\right) & \text { for } x>\frac{1}{4}\end{cases} \\
I^{-}(x)=\left\{\begin{array}{ll}
\sqrt{1-4 x}\left(\ln \left(\frac{1-\sqrt{1-4 x}}{2 \sqrt{x}}\right)+i \pi\right) & \text { for } x<\frac{1}{4} \\
-\sqrt{4 x-1} \arccos \left(-\frac{1}{2 \sqrt{x}}\right) & \text { for } x>\frac{1}{4}
\end{array} .\right.
\end{gathered}
$$

$g(x)$ is given in Eq. (22) and has the special value $g(1)=\frac{\pi}{2 \sqrt{3}}$.

The second form factor is

$$
\begin{aligned}
\tilde{F}^{W g}= & \frac{1}{s_{W}}\left\{-\frac{3 i}{2}\left|V_{t b}\right|^{2}\left[m_{t}^{2} \eta_{U} e^{i \alpha_{U}} C_{0}\left(0, m_{I}^{2}, m_{W}^{2}, m_{t}^{2}, m_{t}^{2}, m_{b}^{2}\right)\right.\right. \\
& \left.\left.+m_{b}^{2} \eta_{D} e^{-i \alpha_{D}} C_{0}\left(0, m_{I}^{2}, m_{W}^{2}, m_{b}^{2}, m_{b}^{2}, m_{t}^{2}\right)\right]\right\}
\end{aligned}
$$

\section{APPENDIX B: $\boldsymbol{H} \rightarrow \boldsymbol{g g}$}

To validate our calculation of the one-loop amplitudes we also compute the well known $H \rightarrow g g[44,45]$. Using the notation,

$$
\mathcal{L}(h g g)=\frac{\alpha_{s}}{4 \pi v}\left(F_{R}^{a} G_{\mu \nu}^{A} G^{B \mu \nu}+F_{R}^{b} \tilde{G}_{\mu \nu}^{A} G^{B \mu \nu}\right) H \delta^{A B}
$$

we find agreement with the well known result,

$$
\begin{aligned}
F_{R}^{a}= & I_{q}\left(\frac{m_{t}^{2}}{m_{H}^{2}}\right)+\frac{3}{4} \frac{v^{2}}{m_{H}^{2}}\left[\left(\lambda_{1}+\lambda_{2}-2 \lambda_{3}\right) I_{s}\left(\frac{m_{I}^{2}}{m_{H}^{2}}\right)\right. \\
& \left.+\left(\lambda_{1}+\lambda_{2}+2 \lambda_{3}\right) I_{s}\left(\frac{m_{R}^{2}}{m_{H}^{2}}\right)+2 \lambda_{1} I_{s}\left(\frac{m_{S^{ \pm}}^{2}}{m_{H}^{2}}\right)\right],
\end{aligned}
$$

and $F_{R}^{b}=0$. In terms of these we have

$$
\Gamma(H \rightarrow g g)=\frac{2}{\pi}\left(\frac{\alpha_{s}}{4 \pi v}\right)^{2} m_{H}^{3}\left(\left|F_{R}^{a}\right|^{2}+\left|F_{R}^{b}\right|^{2}\right) .
$$

[1] A. V. Manohar and M. B. Wise, Flavor changing neutral currents, an extended scalar sector, and the Higgs production rate at the CERN LHC, Phys. Rev. D 74, 035009 (2006).

[2] X.-G. He and G. Valencia, An extended scalar sector to address the tension between a fourth generation and Higgs searches at the LHC, Phys. Lett. B 707, 381 (2012).

[3] B. A. Dobrescu, G. D. Kribs, and A. Martin, Higgs underproduction at the LHC, Phys. Rev. D 85, 074031 (2012).

[4] Y. Bai, J. Fan, and J. L. Hewett, Hiding a heavy Higgs boson at the $7 \mathrm{TeV}$ LHC, J. High Energy Phys. 08 (2012) 014.

[5] G. Cacciapaglia, A. Deandrea, G. D. La Rochelle, and J.-B. Flament, Higgs couplings beyond the Standard Model, J. High Energy Phys. 03 (2013) 029.

[6] J. Cao, P. Wan, J. M. Yang, and J. Zhu, The SM extension with color-octet scalars: Diphoton enhancement and global fit of LHC Higgs data, J. High Energy Phys. 08 (2013) 009.

[7] X.-G. He, Y. Tang, and G. Valencia, Interplay between new physics in one-loop Higgs couplings and the top-quark Yukawa coupling, Phys. Rev. D 88, 033005 (2013).

[8] M. I. Gresham and M. B. Wise, Color octet scalar production at the LHC, Phys. Rev. D 76, 075003 (2007).

[9] C. P. Burgess, M. Trott, and S. Zuberi, Light octet scalars, a heavy Higgs and minimal flavour violation, J. High Energy Phys. 09 (2009) 082.

[10] B. Grinstein, A. L. Kagan, J. Zupan, and M. Trott, Flavor symmetric sectors and collider physics, J. High Energy Phys. 10 (2011) 072.

[11] X.-D. Cheng, X.-Q. Li, Y.-D. Yang, and X. Zhang, $B_{s, d}-$ $\bar{B}_{s, d}$ mixings and $B_{s, d} \rightarrow \ell^{+} \ell^{-}$decays within the ManoharWise model, J. Phys. G 42, 125005 (2015). 
[12] R. Martinez and G. Valencia, Top and bottom tensor couplings from a color octet scalar, Phys. Rev. D 95, 035041 (2017).

[13] T. Faber, Y. Liu, W. Porod, M. Hudec, M. Malinský, F. Staub, and H. Kolešová, Collider phenomenology of a unified leptoquark model, Phys. Rev. D 101, 095024 (2020).

[14] M. Reece, Vacuum instabilities with a wrong-sign HiggsGluon-Gluon amplitude, New J. Phys. 15, 043003 (2013).

[15] X.-G. He, H. Phoon, Y. Tang, and G. Valencia, Unitarity and vacuum stability constraints on the couplings of color octet scalars, J. High Energy Phys. 05 (2013) 026.

[16] L. Cheng and G. Valencia, Two Higgs doublet models augmented by a scalar colour octet, J. High Energy Phys. 09 (2016) 079.

[17] L. Cheng and G. Valencia, Validity of two Higgs doublet models with a scalar color octet up to a high energy scale, Phys. Rev. D 96, 035021 (2017).

[18] M. Gerbush, T. J. Khoo, D. J. Phalen, A. Pierce, and D. Tucker-Smith, Color-octet scalars at the CERN LHC, Phys. Rev. D 77, 095003 (2008).

[19] J. M. Arnold and B. Fornal, Color octet scalars and high pT four-jet events at LHC, Phys. Rev. D 85, 055020 (2012).

[20] X.-G. He, G. Valencia, and H. Yokoya, Color-octet scalars and potentially large $C P$ violation at the LHC, J. High Energy Phys. 12 (2011) 030.

[21] G. D. Kribs and A. Martin, Enhanced di-Higgs production through light colored scalars, Phys. Rev. D 86, 095023 (2012).

[22] A. Hayreter and G. Valencia, LHC constraints on color octet scalars, Phys. Rev. D 96, 035004 (2017).

[23] A. Belyaev, G. Cacciapaglia, H. Cai, G. Ferretti, T. Flacke, A. Parolini, and H. Serodio, Di-boson signatures as standard candles for partial compositeness, J. High Energy Phys. 01 (2017) 094.

[24] L. M. Carpenter and R. Colburn, Searching for standard model adjoint scalars with diboson resonance signatures, J. High Energy Phys. 12 (2015) 151.

[25] L. M. Carpenter, T. Murphy, and M. J. Smylie, Exploring color-octet scalar parameter space in minimal $R$-symmetric models, J. High Energy Phys. 11 (2020) 024.

[26] T. Aaltonen et al. (CDF Collaboration), Invariant Mass Distribution of Jet Pairs Produced in Association with a $W$ boson in $p \bar{p}$ Collisions at $\sqrt{s}=1.96 \mathrm{TeV}$, Phys. Rev. Lett. 106, 171801 (2011).

[27] L. M. Carpenter and S. Mantry, Color-octet, electroweakdoublet scalars and the CDF dijet anomaly, Phys. Lett. B 703, 479 (2011).

[28] T. Enkhbat, X.-G. He, Y. Mimura, and H. Yokoya, Colored scalars and the CDF $W+$ dijet excess, J. High Energy Phys. 02 (2012) 058.
[29] G. Cacciapaglia, A. Deandrea, T. Flacke, and A. Iyer, Gluon-photon signatures for color octet at the LHC (and beyond), J. High Energy Phys. 05 (2020) 027.

[30] N. D. Christensen, P. de Aquino, C. Degrande, C. Duhr, B. Fuks, M. Herquet, F. Maltoni, and S. Schumann, A Comprehensive approach to new physics simulations, Eur. Phys. J. C 71, 1541 (2011).

[31] A. Alloul, N. D. Christensen, C. Degrande, C. Duhr, and B. Fuks, FeynRules 2.0-A complete toolbox for tree-level phenomenology, Comput. Phys. Commun. 185, 2250 (2014).

[32] T. Hahn, Generating Feynman diagrams and amplitudes with FeynArts 3, Comput. Phys. Commun. 140, 418 (2001).

[33] T. Hahn and M. Perez-Victoria, Automatized one loop calculations in four-dimensions and $D$-dimensions, Comput. Phys. Commun. 118, 153 (1999).

[34] R. Mertig, M. Bohm, and A. Denner, FEYN CALC: Computer algebraic calculation of Feynman amplitudes, Comput. Phys. Commun. 64, 345 (1991).

[35] V. Shtabovenko, R. Mertig, and F. Orellana, New developments in FeynCalc 9.0, Comput. Phys. Commun. 207, 432 (2016).

[36] V. Shtabovenko, FeynHelpers: Connecting Feyncalc to FIRE and package-X, Comput. Phys. Commun. 218, 48 (2017).

[37] H. H. Patel, Package-X: A Mathematica package for the analytic calculation of one-loop integrals, Comput. Phys. Commun. 197, 276 (2015).

[38] C.-S. Chen, C.-Q. Geng, D. Huang, and L.-H. Tsai, New scalar contributions to $h \rightarrow Z \gamma$, Phys. Rev. D 87, 075019 (2013).

[39] H. Pois, T. J. Weiler, and T. C. Yuan, Higgs boson decay to four fermions including a single top quark below $t \bar{t}$ threshold, Phys. Rev. D 47, 3886 (1993).

[40] V. Miralles and A. Pich, LHC bounds on colored scalars, Phys. Rev. D 100, 115042 (2019).

[41] L. Cheng, O. Eberhardt, and C. W. Murphy, New theoretical constraints on scalar color octet models, Chin. Phys. C 43, 093101 (2019).

[42] A. M. Sirunyan et al. (CMS Collaboration), Search for pairproduced resonances decaying to quark pairs in protonproton collisions at $\sqrt{s}=13 \mathrm{TeV}$, Phys. Rev. D 98, 112014 (2018).

[43] M. Aaboud et al. (ATLAS Collaboration), A search for pairproduced resonances in four-jet final states at $\sqrt{s}=13 \mathrm{TeV}$ with the ATLAS detector, Eur. Phys. J. C 78, 250 (2018).

[44] J. F. Gunion, H. E. Haber, G. L. Kane, and S. Dawson, The Higgs hunter's guide, Front. Phys. 80, 1 (2000).

[45] A. Djouadi, The Anatomy of electro-weak symmetry breaking. I: The Higgs boson in the standard model, Phys. Rep. 457, 1 (2008). 